\title{
Log-concavity and strong $q$-log-convexity for Riordan arrays and recursive matrices
}

\author{
Bao-Xuan Zhu \\ School of Mathematics and Statistics, Jiangsu Normal University, \\ Xuzhou 221116, People's Republic of China (bxzhu@jsnu.edu.cn)
}

(MS received 29 May 2015; accepted 24 March 2016)

Let $\left[A_{n, k}\right]_{n, k} \geqslant 0$ be an infinite lower triangular array satisfying the recurrence

$$
A_{n, k}=A_{n-1, k-1}+g_{k} A_{n-1, k}+h_{k} A_{n-1, k+1}
$$

for $n \geqslant 1$ and $k \geqslant 0$, where $A_{0,0}=1, A_{0, k}=A_{k,-1}=0$ for $k>0$. We present some criteria for the log-concavity of rows and strong $q$-log-convexity of generating functions of rows. Our results can be applied to many well-known triangular arrays, such as the Pascal triangle, the Stirling triangle of the second kind, the Bell triangle, the large Schröder triangle, the Motzkin triangle, and the Catalan triangles of Aigner and Shapiro, in a unified approach. In addition, we prove that the binomial transformation not only preserves the strong $q$-log-convexity property, but also preserves the strong $q$-log-concavity property. Finally, we demonstrate that the strong $q$-log-convexity property is preserved by the Stirling transformation and Whitney transformation of the second kind, which extends some known results for the strong $q$-log-convexity property.

Keywords: Riordan array; recursive matrix; log-convexity; log-concavity; strong $q$-log-convexity

2010 Mathematics subject classification: Primary 05A10; 05A20; 11B73; 11B83; 15B36

\section{Introduction}

The object of this paper is to study the log-concavity and the strong $q$-log-convexity of sequences arising in some triangular arrays in a unified approach.

For a combinatorial sequence, one often considers its log-concavity or log-convexity. Recall that a sequence of non-negative real numbers $\left(a_{k}\right)_{k \geqslant 0}$ is called concave (respectively, convex) if $a_{k-1}+a_{k+1} \leqslant 2 a_{k}$ (respectively, $a_{k-1}+a_{k+1} \geqslant 2 a_{k}$ ) for $k \geqslant$ 1. It is called log-concave (respectively, log-convex) if $a_{k-1} a_{k+1} \leqslant a_{k}^{2}$ (respectively, $\left.a_{k-1} a_{k+1} \geqslant a_{k}^{2}\right)$ for all $k \geqslant 1$, which is equivalent to having that $a_{n-1} a_{m+1} \leqslant a_{n} a_{m}$ (respectively, $a_{n-1} a_{m+1} \geqslant a_{n} a_{m}$ ) for all $1 \leqslant n \leqslant m$. By the arithmetic-geometric mean inequality, the log-convexity implies the convexity and the concavity implies the log-concavity. In addition, the log-concavity and log-convexity problems occur naturally in combinatorics, analysis, algebra, geometry, probability and statistics, and in many other branches of mathematics and have been extensively investigated; see Stanley's survey article [38] and Brenti's supplement [14] for log-concavity, and Liu and Wang [31] for log-convexity. 
For two polynomials with real coefficients $f(q)$ and $g(q)$, write $f(q) \geqslant_{q} g(q)$ if the difference $f(q)-g(q)$ has only non-negative coefficients. A polynomial sequence $\left(f_{n}(q)\right)_{n \geqslant 0}$ is called $q$-log-concave if

$$
f_{n}(q)^{2} \geqslant_{q} f_{n+1}(q) f_{n-1}(q)
$$

for $n \geqslant 1$. It is called strongly $q$-log-concave if

$$
f_{n}(q) f_{m}(q) \geqslant_{q} f_{n+1}(q) f_{m-1}(q)
$$

for any $m \geqslant n \geqslant 1$. It is called $q$-log-convex if

$$
f_{n+1}(q) f_{n-1}(q) \geqslant_{q} f_{n}(q)^{2}
$$

for $n \geqslant 1$. It is called strongly $q$-log-convex if

$$
f_{n+1}(q) f_{m-1}(q) \geqslant_{q} f_{n}(q) f_{m}(q)
$$

for any $m \geqslant n \geqslant 1$. Clearly, the strong $q$-log-concavity (respectively, strong $q$-logconvexity) implies the $q$-log-concavity (respectively, $q$-log-convexity). In addition, a log-concave (respectively, log-convex) sequence is a special strongly $q$-log-concave (respectively, strongly $q$-log-convex) sequence. The $q$-log-concavity of polynomials has been extensively studied. See, for example, $[15,28,29,34]$. The reader is referred to $[16-18,31,42,43]$ for the strong $q$-log-convexity property.

It is known that there are many triangular arrays in combinatorics. The following are some classical examples.

EXAMPle 1.1.

(1) The Pascal triangle is

$$
P=\left[\left(\begin{array}{l}
n \\
k
\end{array}\right)\right]_{n, k \geqslant 0}=\left[\begin{array}{cccccc}
1 & & & & \\
1 & 1 & & & \\
1 & 2 & 1 & & \\
1 & 3 & 3 & 1 & \\
1 & 4 & 6 & 4 & 1 \\
\vdots & & & & \ddots
\end{array}\right]
$$

where $\left(\begin{array}{c}n+1 \\ k\end{array}\right)=\left(\begin{array}{l}n \\ k\end{array}\right)+\left(\begin{array}{c}n \\ k-1\end{array}\right)$.

(2) The Stirling triangle of the second kind $\left[S_{n, k}\right]_{n, k} \geqslant 0$ satisfies $S_{n+1, k}=S_{n, k-1}+$ $(k+1) S_{n, k}$.

(3) The Catalan triangle of Aigner [3] is

$$
C=\left[C_{n, k}\right]_{n, k \geqslant 0}=\left[\begin{array}{cccccc}
1 & & & & \\
1 & 1 & & & \\
2 & 3 & 1 & & \\
5 & 9 & 5 & 1 & \\
\vdots & & & & \ddots
\end{array}\right],
$$

where $C_{n+1, k}=C_{n, k-1}+2 C_{n, k}+C_{n, k+1}$ and $C_{n+1,0}=C_{n, 0}+C_{n, 1}$. The numbers in the zeroth column are the Catalan numbers $C_{n}$. 
(4) The Catalan triangle of Shapiro [35] is

$$
C^{\prime}=\left[C_{n, k}^{\prime}\right]_{n, k \geqslant 0}=\left[\begin{array}{cccccc}
1 & & & & \\
2 & 1 & & & \\
5 & 4 & 1 & & \\
14 & 14 & 6 & 1 & \\
\vdots & & & & \ddots
\end{array}\right]
$$

where $C_{n+1, k}^{\prime}=C_{n, k-1}^{\prime}+2 C_{n, k}^{\prime}+C_{n, k+1}^{\prime}$ for $k \geqslant 0$. The numbers in the zeroth column are the Catalan numbers $C_{n}$.

(5) The Motzkin triangle $[2,3]$ is

$$
M=\left[M_{n, k}\right]_{n, k \geqslant 0}=\left[\begin{array}{cccccc}
1 & & & & \\
1 & 1 & & & \\
2 & 2 & 1 & & \\
4 & 5 & 3 & 1 & \\
\vdots & & & & \ddots
\end{array}\right],
$$

where $M_{n+1, k}=M_{n, k-1}+M_{n, k}+M_{n, k+1}$ and $M_{n+1,0}=M_{n, 0}+M_{n, 1}$. The numbers in the zeroth column are the Motzkin numbers $M_{n}$.

(6) The large Schröder triangle [20] is

$$
s=\left[s_{n, k}\right]_{n, k \geqslant 0}=\left[\begin{array}{cccccc}
1 & & & & \\
2 & 1 & & & \\
6 & 4 & 1 & & \\
22 & 16 & 6 & 1 & \\
\vdots & & & & \ddots
\end{array}\right],
$$

where $s_{n+1, k}=s_{n, k-1}+2 s_{n, k}+2 s_{n, k+1}$ and $s_{n+1,0}=s_{n, 0}+2 s_{n, 1}$. The numbers in the zeroth column are the large Schröder numbers $S_{n}$.

(7) The Bell triangle [4] is

$$
B=\left[B_{n, k}\right]_{n, k \geqslant 0}=\left[\begin{array}{cccccc}
1 & & & & \\
1 & 1 & & & \\
2 & 3 & 1 & & \\
5 & 10 & 6 & 1 & \\
\vdots & & & & \ddots
\end{array}\right],
$$

where $B_{n+1, k}=B_{n, k-1}+(1+k) B_{n, k}+(1+k) B_{n, k+1}$ and $B_{n+1,0}=B_{n, 0}+B_{n, 1}$. The numbers in the zeroth column are the Bell numbers.

In fact, the above triangles can be obtained in a unified approach as follows. Let $\left[A_{n, k}\right]_{n, k \geqslant 0}$ be an infinite lower triangular array defined by the recurrence

$$
A_{n, k}=A_{n-1, k-1}+g_{k} A_{n-1, k}+h_{k} A_{n-1, k+1}
$$


for $n \geqslant 1$ and $k \geqslant 0$, where $A_{0,0}=1, A_{0, k}=A_{k,-1}=0$ for $k>0 .\left[A_{n, k}\right]_{n, k \geqslant 0}$ is called the recursive matrix and $A_{n, 0}$ are called the Catalan-like numbers; see [3,5]. Aigner $[2,3,5,6]$ researched various combinatorial properties of recursive matrices and Hankel matrices of the Catalan-like numbers. Chen et al. [19] considered the total positivity of recursive matrices. In [42], Zhu gave a criterion for log-convexity of the Catalan-like numbers, i.e. the first column of the recursive matrices $\left[A_{n, k}\right]_{0 \leqslant k \leqslant n}$. Motivated by this, we will show one criterion for log-concavity of each row of recursive matrices; see theorem 2.1. As applications, we prove the log-concavity of row sequences in many well-known triangular arrays, such as the Pascal triangle, the Stirling triangle of the second kind, the Bell triangle, the large Schröder triangle, the Motzkin triangle, and the Catalan triangles of Aigner and Shapiro, in a unified approach.

If $g_{k}=g$ and $h_{k}=h$ for $k \geqslant 0$, then the recursive matrix turns out to be a kind of special interesting Riordan array. Recall that the Riordan array, denoted by $(g(x), f(x))=\left[R_{n, k}\right]_{n, k \geqslant 0}$, is an infinite lower triangular matrix whose generating function of the $k$ th column is $x^{k} f^{k}(x) g(x)$ for $k \geqslant 0$, where $g(0)=1$ and $f(0) \neq 0$. It can also be characterized by two sequences $\left(a_{n}\right)_{n \geqslant 0}$ and $\left(z_{n}\right)_{n \geqslant 0}$ such that

$$
R_{0,0}=1, \quad R_{n+1,0}=\sum_{j \geqslant 0} z_{j} R_{n, j}, R_{n+1, k+1}=\sum_{j \geqslant 0} a_{j} R_{n, k+j}
$$

for $n, k \geqslant 0$. The literature about Riordan arrays is vast and still growing, and the applications cover a wide range of subjects, such as enumerative combinatorics, combinatorial sums, recurrence relations and computer science, among other topics $[19,20,25,26,32,33,36,37]$. Let $\left[T_{n, k}\right]_{n, k} \geqslant 0$ be an array of non-negative numbers satisfying the recurrence relation

$$
T_{n, k}=\left(A n+a_{1} k^{2}+a_{2} k+a_{3}\right) T_{n-1, k}+\left(B n+b_{1} k^{2}+b_{2} k+b_{3}\right) T_{n-1, k-1},
$$

where $T_{n, k}=0$ unless $0 \leqslant k \leqslant n$. Two known results for the strong $q$-log-convexity of the generating functions of row sequences of $\left[T_{n, k}\right]_{n, k \geqslant 0}$, for $a_{1}=b_{1}=0$ and for $A=B=0$, respectively, were proved by Chen et al. [18] and Zhu [43]. Therefore, we also consider a similar problem for the above special interesting Riordan arrays (see theorem 3.4). As consequences, we get the strong $q$-log-convexity of polynomials arising in many well-known triangular arrays, such as the Bell triangle, the large Schröder triangle, the Motzkin triangle, and the Catalan triangles of Aigner and Shapiro, in a unified manner.

A good way of obtaining the log-concavity or log-convexity is via some operators. For instance, Davenport and Pólya [22] proved that the log-convexity is preserved under the binomial convolution. The binomial transformation $y_{n}=\sum_{k=0}^{n}\left(\begin{array}{l}n \\ k\end{array}\right) x_{k}$ preserves the log-concavity property (see, for instance, $[13,27]$ ). More generally, Wang and Yeh [41] showed that the log-concavity property is preserved under the binomial convolution. Ahmia and Belbachir [1] demonstrated that the log-convexity property is preserved under the ordinary multinomials convolution. However, there are few results about the linear transformation preserving the strong $q$-log-convexity property. Motivated by this, in $\S 4$ we research some linear transformations that preserve the strong $q$-log-convexity property. We prove that the binomial transformation not only preserves the strong $q$-log-convexity property, but also preserves 
the strong $q$-log-concavity property. We also demonstrate that the strong $q$-logconvexity is preserved by the Stirling transformation and Whitney transformation of the second kind, which extends some known results for the strong $q$-log-convexity.

\section{Log-concavity of the rows}

Many sequences of binomial coefficients share various log-concavity properties. For example, each row sequence in the Pascal triangle is log-concave, which is one basic and classical result. More generally, any sequence of integers lying along a finite ray in the Pascal triangle is log-concave (see $[7,8,39]$ ). It is also known that each row sequence in the Stirling triangle of the second kind is log-concave (see, for instance, [24,30]). Recently, Belbachir and Tebtoub [9] also proved that every row sequence in the 2-successive associated Stirling triangle is log-concave. Thus, in this section, we will give a sufficient condition for the log-concavity of rows in recursive matrices.

THEOREM 2.1. Let an infinite lower triangular array $\left[A_{n, k}\right]_{n, k \geqslant 0}$ satisfy the recurrence

$$
A_{n, k}=A_{n-1, k-1}+g_{k} A_{n-1, k}+h_{k} A_{n-1, k+1}
$$

for $n \geqslant 1$ and $k \geqslant 0$, where $A_{0,0}=1, A_{0, k}=A_{0,-1}=0$ for $k>0$. Assume that non-negative sequences $\left(g_{k}\right)_{k \geqslant 0}$ and $\left(h_{k}\right)_{k \geqslant 0}$ are both concave. If

$$
2 g_{k} h_{k} \geqslant g_{k+1} h_{k-1}+g_{k-1} h_{k+1} \quad \text { and } \quad g_{k+1} g_{k-1} \geqslant h_{k-1}
$$

for all $k \geqslant 1$, then, for any fixed $n$, the row sequence $\left(A_{n, k}\right)_{0 \leqslant k \leqslant n}$ is log-concave in $k$.

Proof. To show that $\left(A_{n, k}\right)_{0 \leqslant k \leqslant n}$ is log-concave in $k$, it suffices to prove that

$$
A_{n, k}^{2}-A_{n, k+1} A_{n, k-1} \geqslant 0
$$

for any $k \geqslant 0$, which will be done by induction on $n$. It is clear for $n=0$. Thus, we suppose that it follows for $1 \leqslant n \leqslant m-1$. Then for $n=m$ and $0 \leqslant k \leqslant m$ we have that

$$
\begin{aligned}
& A_{m, k}^{2}-A_{m, k+1} A_{m, k-1} \\
& =\left[A_{m-1, k-1}+g_{k} A_{m-1, k}+h_{k} A_{m-1, k+1}\right]^{2} \\
& -\left[A_{m-1, k}+g_{k+1} A_{m-1, k+1}+h_{k+1} A_{m-1, k+2}\right] \\
& \times\left[A_{m-1, k-2}+g_{k-1} A_{m-1, k-1}+h_{k-1} A_{m-1, k}\right] \\
& =\underbrace{A_{m-1, k-1}^{2}-A_{m-1, k} A_{m-1, k-2}}+\left[2 g_{k}-g_{k-1}\right] A_{m-1, k-1} A_{m-1, k} \\
& -g_{k+1} A_{m-1, k+1} A_{m-1, k-2}+\underbrace{h_{k}^{2} A_{m-1, k+1}^{2}-h_{k+1} h_{k-1} A_{m-1, k+2} A_{m-1, k}} \\
& +\left[2 h_{k} g_{k}-h_{k-1} g_{k+1}\right] A_{m-1, k} A_{m-1, k+1}-h_{k+1} g_{k-1} A_{m-1, k+2} A_{m-1, k-1} \\
& +\underbrace{g_{k}^{2} A_{m-1, k}^{2}-g_{k+1} g_{k-1} A_{m-1, k+1} A_{m-1, k-1}} \\
& +\left[2 h_{k} A_{m-1, k-1} A_{m-1, k+1}-h_{k+1} A_{m-1, k+2} A_{m-1, k-2}-h_{k-1} A_{m-1, k}^{2}\right] .
\end{aligned}
$$


In the following, we will consider the non-negativity of (2.2). On the one hand, it follows from the log-concavity of $\left(A_{m-1, k}\right)_{0 \leqslant k \leqslant m-1}$ that we have

$$
A_{m-1, k-1}^{2}-A_{m-1, k} A_{m-1, k-2} \geqslant 0 .
$$

In addition, we know that $\left(g_{k}\right)_{k \geqslant 0}$ and $\left(h_{k}\right)_{k \geqslant 0}$ are log-concave by the concavities of $\left(g_{k}\right)_{k \geqslant 0}$ and $\left(h_{k}\right)_{k \geqslant 0}$. Thus, we also have

$$
h_{k}^{2} A_{m-1, k+1}^{2}-h_{k+1} h_{k-1} A_{m-1, k+2} A_{m-1, k} \geqslant 0 .
$$

On the other hand, by the log-concavity of $\left(A_{m-1, k}\right)_{0 \leqslant k \leqslant m-1}$ and the given concave condition $2 g_{k} \geqslant g_{n, k-1}+g_{k+1}$, we deduce that

$$
\begin{aligned}
{\left[2 g_{k}-g_{k-1}\right] A_{m-1, k-1} A_{m-1, k}-g_{k+1} } & A_{m-1, k+1} A_{m-1, k-2} \\
& \geqslant\left[2 g_{k}-g_{k-1}-g_{k+1}\right] A_{m-1, k-1} A_{m-1, k} \\
& \geqslant 0 .
\end{aligned}
$$

By a similar method, we get

$$
\begin{aligned}
{\left[2 h_{k} g_{k}-h_{k-1} g_{k+1}\right] A_{m-1, k} } & A_{m-1, k+1}-h_{k+1} g_{k-1} A_{m-1, k+2} A_{m-1, k-1} \\
& \geqslant\left[2 h_{k} g_{k}-h_{k-1} g_{k+1}-h_{k+1} g_{k-1}\right] A_{m-1, k} A_{m-1, k+1} \\
& \geqslant 0 .
\end{aligned}
$$

Finally, by $2 h_{k} \geqslant h_{k+1}+h_{k-1}$ and the log-concavity of $\left(g_{k}\right)_{k \geqslant 0}$, we obtain that

$$
\begin{aligned}
& \underbrace{g_{k}^{2} A_{m-1, k}^{2}-g_{k+1} g_{k-1} A_{m-1, k+1} A_{m-1, k-1}}+2 h_{k} A_{m-1, k-1} A_{m-1, k+1} \\
& \text { - } h_{k+1} A_{m-1, k+2} A_{m-1, k-2}-h_{k-1} A_{m-1, k}^{2} \\
& \geqslant \underbrace{g_{k}^{2} A_{m-1, k}^{2}-g_{k+1} g_{k-1} A_{m-1, k+1} A_{m-1, k-1}} \\
& +\left(h_{k+1}+h_{k-1}\right) A_{m-1, k-1} A_{m-1, k+1}-h_{k+1} A_{m-1, k+2} A_{m-1, k-2} \\
& -h_{k-1} A_{m-1, k}^{2} \\
& \geqslant \underbrace{g_{k+1} g_{k-1}\left[A_{m-1, k}^{2}-A_{m-1, k+1} A_{m-1, k-1}\right]} \\
& +h_{k-1}\left[A_{m-1, k-1} A_{m-1, k+1}-A_{m-1, k}^{2}\right] \\
& +h_{k+1}\left[A_{m-1, k+1} A_{m-1, k-1}-A_{m-1, k+2} A_{m-1, k-2}\right] \\
& =\underbrace{\left[g_{k+1} g_{k-1}-h_{k-1}\right]\left[A_{m-1, k}^{2}-A_{m-1, k+1} A_{m-1, k-1}\right]} \\
& +h_{k+1}\left[A_{m-1, k+1} A_{m-1, k-1}-A_{m-1, k+2} A_{m-1, k-2}\right] \\
& \geqslant 0 \text {, }
\end{aligned}
$$

where the last inequality follows from the log-concavity of $\left(A_{m-1, k}\right)_{0 \leqslant k \leqslant m-1}$ and $g_{k+1} g_{k-1} \geqslant h_{k-1}$.

Thus, by (2.2)-(2.7), we demonstrate that

$$
A_{m, k}^{2}-A_{m, k+1} A_{m, k-1} \geqslant 0
$$

for $0 \leqslant k \leqslant m$. This completes the proof. 
The following special case may be more interesting.

Corollary 2.2. Define the matrix $\left[A_{n, k}\right]_{n, k \geqslant 0}$ recursively:

$$
\begin{aligned}
& A_{0,0}=1, \\
& A_{0, k}=0 \quad(k>0), \\
& A_{n, 0}=e A_{n-1,0}+h A_{n-1,1}, \\
& A_{n, k}=A_{n-1, k-1}+g A_{n-1, k}+h A_{n-1, k+1} \quad(n \geqslant 1) .
\end{aligned}
$$

If eg $\geqslant h \geqslant 0$ and $g \geqslant e \geqslant 0$, then for any fixed $n$ each row $\left(A_{n, k}\right)_{0 \leqslant k \leqslant n}$ is log-concave.

Applying our results to some combinatorial arrays in example 1.1, we have the following result.

Proposition 2.3. Each row sequence in the Pascal triangle, the Stirling triangle of the second kind, the Catalan triangles of Aigner and Shapiro, the Motzkin triangle, the large Schröder triangle and the Bell triangle is log-concave.

\section{Strong $q$-log-convexity of generating functions of rows}

In this section we present a criterion for the strong $q$-log-convexity of generating functions of rows in certain Riordan arrays.

Recall that a matrix $M=\left(m_{i j}\right)_{i, j \geqslant 0}$ of non-negative numbers is said to be 2 -order totally positive ( $\mathrm{TP}_{2}$ for short) if all of its minors of order 1 and 2 are non-negative. We often find that the entries of a matrix are polynomials with real coefficients in an independent variable $q$. Similarly, if any corresponding minor of order 1 or 2 is a polynomial with non-negative coefficients in $q$, then we say that it is $q-\mathrm{TP}_{2}$. See Karlin [27] for more details.

The following lemmas will be very useful in our proof.

LEMMA 3.1. A sufficient condition that a matrix is $\mathrm{TP}_{2}$ is that all leading principal submatrices are $T P_{2}$.

Lemma 3.2. If two matrices $\mathcal{A}$ and $\mathcal{B}$ are both $T P_{2}$, then we have that $\mathcal{A B}$ is $T P_{2}$.

By the classical Cauchy-Binet formula, we also have the following lemma.

LEMMA 3.3. If the matrix $\mathcal{A}$ is $T P_{2}$ and the matrix $\mathcal{B}$ is $q-T P_{2}$, then we have that $\mathcal{A B}$ is $q-T P_{2}$.

The next theorem is our result for the strong $q$-log-convexity.

THEOREM 3.4. Assume that the Riordan array $\left[A_{n, k}\right]_{n, k \geqslant 0}$ satisfies the recurrence

$$
\begin{aligned}
& A_{n, k}=A_{n-1, k-1}+g A_{n-1, k}+h A_{n-1, k+1}, \\
& A_{n, 0}=e A_{n-1,0}+h A_{n-1,1}
\end{aligned}
$$

for $n \geqslant 1$ and $k \geqslant 1$, where $A_{0,0}=1, A_{0, k}=0$ for $k>0$. If ge $\geqslant h \geqslant 0$ and $g \geqslant e \geqslant 0$, then the generating functions $A_{n}(q)=\sum_{k \geqslant 0} A_{n, k} q^{k}$ for $n \geqslant 0$ form a strongly q-log-convex sequence. 
Proof. Define a triangular array $\mathcal{S}=\left[b_{n, k}(q)\right]_{n, k \geqslant 0}$, where

$$
b_{n, k}(q)=\sum_{i \geqslant k} A_{n, i} q^{i} \quad \text { for all } n \text { and } k .
$$

Thus, by (3.1), it is not hard to verify that the triangular array $\mathcal{S}=\left[b_{n, k}(q)\right]_{n, k \geqslant 0}$ satisfies the recurrence

$$
\begin{aligned}
& b_{n, k}(q)=q b_{n-1, k-1}(q)+g b_{n-1, k}(q)+\frac{h}{q} b_{n-1, k+1}(q) \quad(n \geqslant 1, k \geqslant 1), \\
& b_{n, 0}(q)=(e+q) b_{n-1,0}(q)+\left(g-e+\frac{h}{q}\right) b_{n-1,1}(q) \quad(n \geqslant 1),
\end{aligned}
$$

where $b_{0,0}=1, b_{0, k}=0$ for $k>0$. It is clear that $A_{n}(q)=b_{n, 0}(q)$.

So, by $(3.2)$ we have

$$
\begin{aligned}
A_{n}(q) A_{m}(q)-A_{n+1}(q) A_{m-1}(q) & \\
= & b_{n, 0}(q) b_{m, 0}(q)-b_{n+1,0}(q) b_{m-1,0}(q) \\
= & b_{n, 0}(q)\left[(e+q) b_{m-1,0}(q)+(g-e+h / q) b_{m-1,1}(q)\right] \\
& \quad-b_{m-1,0}(q)\left[(e+q) b_{n, 0}(q)+(g-e+h / q) b_{n, 1}(q)\right] \\
= & {[(g-e) q+h]\left[b_{n, 0}(q) b_{m-1,1}(q)-b_{m-1,0}(q) b_{n, 1}(q)\right] / q }
\end{aligned}
$$

for any $m \geqslant n$. Thus, in order to prove the strong $q$-log-convexity of $\left(A_{n}(q)\right)_{n \geqslant 0}$, it suffices to prove that the matrix $\mathcal{S}$ is $q-\mathrm{TP}_{2}$ since $g \geqslant e$ and $h \geqslant 0$.

Consider the infinite lower triangular matrix

$$
\mathcal{A}=\left(A_{n, k}\right)_{k, n \geqslant 0}=\left(\begin{array}{ccccc}
A_{0,0} & 0 & 0 & \cdots & 0 \\
A_{1,0} & A_{1,1} & 0 & \cdots & 0 \\
A_{2,0} & A_{2,1} & A_{2,2} & \cdots & 0 \\
\vdots & \vdots & \vdots & \ddots & \vdots
\end{array}\right)
$$

and an infinite lower triangular matrix $\mathcal{E}=\left(q^{i}\right)_{i \geqslant j \geqslant 0}$. It is clear that $\mathcal{S}=\mathcal{A} \mathcal{E}$ and $\mathcal{E}$ is $q-\mathrm{TP}_{2}$, which implies that $\mathcal{S}$ is $q-\mathrm{TP}_{2}$ by lemma 3.3 if $\mathcal{A}$ is $\mathrm{TP}_{2}$. Thus, in what follows we will prove that $\mathcal{A}$ is $\mathrm{TP}_{2}$.

Define an infinite matrix

$$
\mathcal{J}=\left(\begin{array}{ccccccc}
e & 1 & 0 & 0 & 0 & \cdots & 0 \\
h & g & 1 & 0 & 0 & \cdots & 0 \\
0 & h & g & 1 & 0 & \cdots & 0 \\
0 & 0 & h & g & 1 & \cdots & 0 \\
\vdots & \vdots & \vdots & \vdots & \vdots & \ddots & \vdots
\end{array}\right)
$$

Define the matrix $\mathcal{Q}$ obtained from $\mathcal{A}$ by deleting its first row. Assume that the $n$th leading principal submatrices of $\mathcal{A}, \mathcal{Q}$ and $\mathcal{J}$ are $\mathcal{A}_{n}, \mathcal{S}_{n}$ and $\mathcal{J}_{n}$, respectively. Thus, by (3.1) we have

$$
\mathcal{Q}_{n}=\mathcal{A}_{n} \mathcal{J}_{n}
$$

Note that $\mathcal{J}$ is $\mathrm{TP}_{2}$ since $g^{2} \geqslant h$ and $g e \geqslant h$. Thus, by induction on $n$, we conclude that $\mathcal{A}_{n}$ is $\mathrm{TP}_{2}$ by lemma 3.2 . So, by lemma 3.1 we have that $\mathcal{A}$ is $\mathrm{TP}_{2}$. This completes the proof. 
It is clear that theorem 3.4 can be applied to some of the triangular arrays in example 1.1 in a unified manner.

Proposition 3.5. The generating functions of rows in Catalan triangles of Aigner and Shapiro, the Motzkin triangle, and the large Schröder triangle respectively form strongly q-log-convex sequences.

\section{Strong $q$-log-convexity and linear transformations}

It is known that many polynomials, denoted by $A_{n}(q)=\sum_{k=0}^{n} A_{n, k} q^{k}$ for $n \geqslant 0$, have strong $q$-log-convexity (see $[18,42]$ ). Note that the sequence $\left\{q^{k}\right\}_{k \geqslant 0}$ is strongly $q$-log-convex. So it is natural to consider the strong $q$-log-convexity of the linear transformation

$$
g_{n}(q)=\sum_{k=0}^{n} A_{n, k} f_{k}(q)
$$

for $n \geqslant 0$ if $\left(f_{k}(q)\right)_{k \geqslant 0}$ is strongly $q$-log-convex. In this section we will study some linear transformations that preserve the strong $q$-log-convexity property.

THEOREM 4.1. Assume that $\left(a_{n, k}\right)_{0 \leqslant k \leqslant n}$ and $\left(b_{n, k}\right)_{0 \leqslant k \leqslant n}$ are two triangles of nonnegative numbers. If the linear transformations $t_{n}=\sum_{k=0}^{n} a_{n, k} f_{k}(q)$ and $s_{n}=$ $\sum_{k=0}^{n} b_{n, k} f_{k}(q)$ preserve the strong $q$-log-convexity property, then so does

$$
g_{n}(q)=\sum_{k=0}^{n} T_{n, k} f_{k}(q)
$$

for $n \geqslant 0$, where $T_{n, k}=\sum_{j=k}^{n} a_{n, j} b_{j, k}$.

Proof. Let $\left(f_{k}(q)\right)_{n \geqslant 0}$ be a strongly $q$-log-convex sequence. Notice that

$$
g_{n}(q)=\sum_{k=0}^{n} f_{k}(q) \sum_{j=k}^{n} a_{n, j} b_{j, k}=\sum_{j=0}^{n} a_{n, j}\left[\sum_{k=0}^{j} b_{j, k} f_{k}(q)\right] .
$$

Let $h_{j}(q)=\sum_{k=0}^{j} b_{j, k} f_{k}(q)$ for $0 \leqslant j \leqslant n$, which is strongly $q$-log-convex since $s_{n}=\sum_{k=0}^{n} b_{n, k} f_{k}(q)$ preserves the strong $q$-log-convexity property. Thus, it follows from the linear transformations $t_{n}=\sum_{k=0}^{n} a_{n, k} f_{k}(q)$ that preserve the strong $q$-logconvexity property that $\left(g_{k}(q)\right)_{k \geqslant 0}$ is strongly $q$-log-convex. The proof is complete.

THEOREM 4.2. The binomial transformation $g_{n}(q)=\sum_{k=0}^{n}\left(\begin{array}{l}n \\ k\end{array}\right) f_{k}(q)$ preserves the strong q-log-convexity property.

Proof. Let $\left(f_{k}(q)\right)_{k \geqslant 0}$ be a strongly $q$-log-convex sequence. We first claim that $\left(f_{k}(q)+f_{k+1}(q)\right)_{k \geqslant 0}$ is strongly $q$-log-convex, which follows from

$$
\begin{aligned}
{\left[f_{j-1}(q)+\right.} & \left.f_{j-2}(q)\right]\left[f_{i+1}(q)+f_{i}(q)\right]-\left[f_{j}(q)+f_{j-1}(q)\right]\left[f_{i}(q)+f_{i-1}(q)\right] \\
= & {\left[f_{j-1}(q) f_{i+1}(q)-f_{j}(q) f_{i}(q)\right]+\left[f_{j-2}(q) f_{i}(q)-f_{j-1}(q) f_{i-1}(q)\right] } \\
& \quad+\left[f_{j-2}(q) f_{i+1}(q)-f_{j}(q) f_{i-1}(q)\right] \\
\geqslant & \geqslant
\end{aligned}
$$

for any $i \geqslant j \geqslant 0$ since $\left(f_{k}(q)\right)_{k \geqslant 0}$ is strongly $q$-log-convex. 
Using this claim, we will prove the desired result by induction on $n$. If $0 \leqslant n \leqslant 3$, then we have

$$
\begin{aligned}
& g_{0}(q)=f_{0}(q), \\
& g_{1}(q)=f_{0}(q)+f_{1}(q), \\
& g_{2}(q)=f_{0}(q)+2 f_{1}(q)+f_{2}(q), \\
& g_{3}(q)=f_{0}(q)+3 f_{1}(q)+3 f_{2}(q)+f_{3}(q),
\end{aligned}
$$

since $g_{n}(q)=\sum_{k=0}^{n}\left(\begin{array}{l}n \\ k\end{array}\right) f_{k}(q)$. Therefore, it follows from the strong $q$-log-convexity of $\left(f_{k}(q)\right)_{k \geqslant 0}$ that

$$
\begin{aligned}
g_{2}(q) g_{0}(q)-g_{1}^{2}(q) & =f_{2}(q) f_{0}(q)-f_{1}^{2}(q) \geqslant_{q} 0 \\
g_{3}(q) g_{0}(q)-g_{2}(q) g_{1}(q) & =2\left[f_{2}(q) f_{0}(q)-f_{1}^{2}(q)\right]+\left[f_{0}(q) f_{3}(q)-f_{1}(q) f_{2}(q)\right] \geqslant_{q} 0 \\
g_{3}(q) g_{1}(q)-g_{2}^{2}(q) & =\left[f_{2}(q) f_{0}(q)-f_{1}^{2}(q)\right]+\left[f_{0}(q) f_{3}(q)-f_{1}(q) f_{2}(q)\right] \\
& +\left[f_{3}(q) f_{1}(q)-f_{2}^{2}(q)\right] \geqslant_{q} 0
\end{aligned}
$$

which imply that $g_{0}(q), g_{1}(q), g_{2}(q), g_{3}(q)$ is strongly $q$-log-convex. So we proceed to the inductive step $(n \geqslant 4)$.

Note that

$$
\begin{aligned}
g_{n}(q) & =\sum_{k=0}^{n}\left(\begin{array}{l}
n \\
k
\end{array}\right) f_{k}(q) \\
& =\sum_{k=0}^{n-1}\left(\begin{array}{c}
n-1 \\
k
\end{array}\right)\left(f_{k}(q)+f_{k+1}(q)\right) .
\end{aligned}
$$

Therefore, by the induction hypothesis and the strong $q$-log-convexity of $\left(f_{k}(q)+\right.$ $\left.f_{k+1}(q)\right)_{k \geqslant 0}$, we have that $g_{0}(q), g_{1}(q), g_{2}(q), \ldots, g_{n}(q)$ is strongly $q$-log-convex.

The Bell polynomials are given by $B_{n+1}(q)=\sum_{k \geqslant 0} S_{n+1, k} x^{k}$, where $S_{n, k}$ are the Stirling numbers of the second kind. It has been shown that the polynomials $B_{n}(q)$ form a strongly $q$-log-convex sequence (see, for instance, [18, 42, 43]). Note that $B_{n+1}(q)=\sum_{k=0}^{n}\left(\begin{array}{l}n \\ k\end{array}\right) B_{n}(q)$. Thus, using theorem 4.2, we can give a new proof for the strong $q$-log-convexity of $B_{n}(q)$.

Similar to the proof of theorem 4.2 , we also have the following result.

TheOREM 4.3. The binomial transformation $g_{n}(q)=\sum_{k=0}^{n}\left(\begin{array}{l}n \\ k\end{array}\right) f_{k}(q)$ preserves the strong q-log-concavity property.

Note that a log-concave sequence is a special strong $q$-log-concave sequence. Thus, theorem 4.3 generalizes the result that the binomial transformation $y_{n}=$ $\sum_{k=0}^{n}\left(\begin{array}{l}n \\ k\end{array}\right) x_{k}$ preserves the log-concavity property (see, for instance, [13,27]).

The following is another criterion for the linear transformation to preserve the strong $q$-log-convexity property. 
TheOREM 4.4. Let $T_{n, k}=\sum_{j=k-1}^{n-1}\left(\begin{array}{c}n-1 \\ j\end{array}\right) T_{j, k-1}$ for $n, k \geqslant 1$ and $T_{n, 0}=T_{0, k}=0$ unless $T_{0,0}=1$. Then the linear transformation

$$
g_{n}(q)=\sum_{k=0}^{n} T_{n, k} f_{k}(q)
$$

preserves the strong q-log-convexity property.

Proof. Let $\left(f_{n}(q)\right)_{n \geqslant 0}$ be a strongly $q$-log-convex sequence. It suffices to prove that the sequence $\left(g_{n}(q)\right)_{n \geqslant 0}$ is $q$-log-convex. We proceed by induction on $n$. It follows from the given conditions that we can obtain

$$
\begin{aligned}
& g_{0}(q)=T_{0,0} f_{0}(q)=f_{0}(q), \\
& g_{1}(q)=T_{1,0} f_{0}(q)+T_{1,1} f_{1}(q)=f_{1}(q), \\
& g_{2}(q)=T_{2,0} f_{0}(q)+T_{2,1} f_{1}(q)+T_{2,2} f_{2}(q)=f_{1}(q)+f_{2}(q), \\
& g_{3}(q)=T_{3,0} f_{0}(q)+T_{3,1} f_{1}(q)+T_{3,2} f_{2}(q)+T_{3,3} f_{3}(q)=f_{1}(q)+3 f_{2}(q)+f_{3}(q) .
\end{aligned}
$$

Thus, we deduce that

$$
\begin{aligned}
g_{2}(q) g_{0}(q)-g_{1}(q)^{2}= & f_{2}(q) f_{0}(q)-f_{1}(q)^{2}+f_{1}(q) f_{0}(q) \geqslant_{q} 0, \\
g_{3}(q) g_{0}(q)-g_{1}(q) g_{2}(q)= & {\left[f_{1}(q)+3 f_{2}(q)+f_{3}(q)\right] f_{0}(q)-f_{1}(q)\left[f_{1}(q)+f_{2}(q)\right] } \\
= & {\left[f_{1}(q) f_{0}(q)+2 f_{2}(q) f_{0}(q)\right]+\left[f_{2}(q) f_{0}(q)-f_{1}(q)^{2}\right] } \\
& \quad+\left[f_{3}(q) f_{0}(q)-f_{1}(q) f_{2}(q)\right] \geqslant_{q} 0, \\
g_{3}(q) g_{1}(q)-g_{2}(q)^{2}= & {\left[f_{1}(q)+3 f_{2}(q)+f_{3}(q)\right] f_{1}(q)-\left[f_{1}(q)+f_{2}(q)\right]^{2} } \\
= & f_{1}(q) f_{2}(q)+f_{1}(q) f_{3}(q)-f_{2}^{2}(q) \geqslant_{q} 0
\end{aligned}
$$

since $\left(f_{n}(q)\right)_{n \geqslant 0}$ is strongly $q$-log-convex. This implies that $g_{0}(q), g_{1}(q), g_{2}(q), g_{3}(q)$ is strongly $q$-log-convex. Now assume that $g_{0}(q), g_{1}(q), g_{2}(q), \ldots, g_{n-1}(q)$ is strongly $q$-log-convex for $n \geqslant 4$, i.e. $g_{j}(q)=\sum_{k=0}^{j} T_{j, k} f_{k}(q)$ preserves the strong $q$-logconvexity property for $0 \leqslant j \leqslant n-1$. Since

$$
T_{n, k}=\sum_{j=k-1}^{n-1}\left(\begin{array}{c}
n-1 \\
j
\end{array}\right) T_{j, k-1},
$$

we have

$$
\begin{aligned}
g_{n}(q) & =\sum_{k=0}^{n} T_{n, k} f_{k}(q) \\
& =\sum_{k=0}^{n} f_{k}(q) \sum_{j=k-1}^{n-1}\left(\begin{array}{c}
n-1 \\
j
\end{array}\right) T_{j, k-1} \\
& =\sum_{j=0}^{n-1}\left(\begin{array}{c}
n-1 \\
j
\end{array}\right) \sum_{k=0}^{j} T_{j, k} f_{k+1}(q) \\
& =\sum_{j=0}^{n-1}\left(\begin{array}{c}
n-1 \\
j
\end{array}\right) h_{j}(q),
\end{aligned}
$$


where

$$
h_{j}(q)=\sum_{k=0}^{j} T_{j, k} f_{k+1}(q) \quad \text { for } 0 \leqslant j \leqslant n-1 .
$$

Then the sequence $h_{0}(q), h_{1}(q), \ldots, h_{n-1}(q)$ is strongly $q$-log-convex by the induction hypothesis, and so is the sequence $g_{0}(q), g_{1}(q), \ldots, g_{n}(q)$ since the linear transformation $t_{n}(q)=\sum_{k=0}^{n}\left(\begin{array}{l}n \\ k\end{array}\right) f_{k}(q)$ preserves the strong $q$-log-convexity property. This completes the proof.

The Stirling numbers $S_{n, k}$ of the second kind are defined by the relation

$$
x^{n}=\sum_{k=0}^{n} S_{n, k}(x)_{k}
$$

for $n \geqslant 0$, where $(x)_{k}=x(x-1)(x-2) \cdots(x-k+1)$. It is known that

$$
S_{n, k}=\sum_{j=k-1}^{n-1}\left(\begin{array}{c}
n-1 \\
j
\end{array}\right) S_{j, k-1},
$$

where $S_{n, 0}=S_{0, k}=0, S_{0,0}=1$; see [21, p. 209]. Hence, by theorem 4.4, we have the next result, which extends the strong $q$-log-convexity of Bell polynomials.

Proposition 4.5. The Stirling transformation of the second kind

$$
g_{n}(q)=\sum_{k=0}^{n} S_{n, k} f_{k}(q)
$$

preserves the strong q-log-convexity property.

In [40], Tanny introduced the following polynomials:

$$
F_{n}(q)=\sum_{k=0}^{n} S_{n, k} k ! q^{k} .
$$

By proposition 4.5, we can give a new proof of the following result.

COROLlary 4.6 (Chen et al. [18]). The polynomials $F_{n}(q)$ form a strongly $q$-logconvex sequence.

The Dowling lattice $Q_{n}(G)$ is a geometric lattice of rank $n$ over a finite group $G$ of order $m$ and has many remarkable properties; see [10-12,23]. When $m=1$, that is, $G$ is the trivial group, $Q_{n}(G)$ is the lattice $\prod_{n+1}$ of partitions of an $(n+1)$ element set. So the Dowling lattices can be viewed as group-theoretic analogues of the partition lattices. Let $W_{m}(n, k)$ be the Whitney numbers of the second kind. It is known that [11]

$$
W_{m}(n, k)=m^{-k} \sum_{i=k}^{n}\left(\begin{array}{l}
n \\
k
\end{array}\right) S_{i, k} m^{i} .
$$

Thus, the following proposition is immediate by theorems 4.1 and 4.2 , and proposition 4.5 . 
Proposition 4.7. The Whitney transformation of the second kind

$$
g_{n}(q)=\sum_{k=0}^{n} W_{m}(n, k) f_{k}(q)
$$

preserves the strong $q$-log-convexity property.

The Dowling polynomials are given by $D_{n}(q)=\sum_{k=0}^{n} W_{m}(n, k) q^{k}$. In addition, Benoumhani [11] introduced the following generalized polynomials: $F_{n, m}(q)=$ $\sum_{k=0}^{n} k ! W_{m}(n, k) m^{k} q^{k}$ and $G_{n, m}(q)=\sum_{k=0}^{n} k ! W_{m}(n, k) q^{k}$. Obviously, proposition 4.7 immediately implies the following result.

COROLlary 4.8 (Chen et al. [18]). The polynomials $D_{n}(q), F_{n, m}(q)$ and $G_{n, m}(q)$ are all strongly $q$-log-convex.

\section{Acknowledgements}

This work was supported partly by the National Natural Science Foundation of China (Grant no. 11571150).

\section{References}

1 M. Ahmia and H. Belbachir. Preserving log-convexity for generalized Pascal triangles. Electron. J. Combin. 19 (2012), paper 16.

$2 \quad$ M. Aigner. Motzkin numbers. Eur. J. Combin. 19 (1998), 663-675.

3 M. Aigner. Catalan-like numbers and determinants. J. Combin. Theory A 87 (1999), 33-51.

4 M. Aigner. A characterization of the Bell numbers. Discr. Math. 205 (1999), 207-210.

5 M. Aigner. Catalan and other numbers: a recurrent theme. In Algebraic combinatorics and computer science (ed. H. Crapo and D. Senato), pp. 347-390 (Springer, 2001).

$6 \quad$ M. Aigner. Enumeration via ballot numbers. Discr. Math. 308 (2008), 2544-2563.

$7 \quad$ H. Belbachir and L. Szalay. Unimodal rays in the ordinary and generalized Pascal triangles. J. Integer Seq. 11 (2008), article 08.2.4.

8 H. Belbachir and L. Szalay. Unimodal rays in the regular and generalized Pascal pyramids. Electron. J. Combin. 18 (2011), paper 79.

9 H. Belbachir and A.-F. Tebtoub. The 2-successive associated Stirling numbers, FibonacciStirling numbers and unimodality. C. R. Acad. Sci. Paris Sér. I 353 (2015), 767-771.

10 M. Benoumhani. On Whitney numbers of Dowling lattices. Discr. Math. 159 (1996), 13-33.

11 M. Benoumhani. On some numbers related to Whitney numbers of Dowling lattices. Adv. Appl. Math. 19 (1997), 106-116.

12 M. Benoumhani. Log-concavity of Whitney numbers of Dowling lattices. Adv. Appl. Math. 22 (1999), 181-189.

13 F. Brenti. Unimodal, log-concave, and Pólya frequency sequences in combinatorics, Memoirs of the American Mathematical Society, vol. 81, no. 413 (Providence, RI: American Mathematical Society, 1989).

14 F. Brenti. Log-concave and unimodal sequences in algebra, combinatorics, and geometry: an update. Contemp. Math. 178 (1994), 71-89.

15 L. M. Butler. The $q$-log concavity of $q$-binomial coeffcients. J. Combin. Theory A 54 (1990), $54-63$.

16 W. Y. C. Chen, L. X. W. Wang and A. L. B. Yang. Schur positivity and the $q$-log-convexity of the Narayana polynomials. J. Algebraic Combin. 32 (2010), 303-338.

17 W. Y. C. Chen, R. L. Tang, L. X. W. Wang and A. L. B. Yang. The $q$-log-convexity of the Narayana polynomials of type B. Adv. Appl. Math. 44 (2010), 85-110.

18 W. Y. C. Chen, L. X. W. Wang and A. L. B. Yang. Recurrence relations for strongly $q$-log-convex polynomials. Can. Math. Bull. 54 (2011), 217-229. 
19 X. Chen, H. Liang and Y. Wang. Total positivity of recursive matrices. Linear Alg. Applic. 471 (2015), 383-393.

20 G.-S. Cheon, H. Kim and L. W. Shapiro. Combinatorics of Riordan arrays with identical A and Z sequences. Discr. Math. 312 (2012), 2040-2049.

21 L. Comtet. Advanced combinatorics (Dordrecht: D. Reidel Publishing Co., 1974).

22 H. Davenport and G. Pólya. On the product of two power series. Can. J. Math. 1 (1949), $1-5$.

23 T. A. Dowling. A class of geometric lattices based on nite groups. J. Combin. Theory B 14 (1973), 61-86. (Erratum. J. Combin. Theory B 15 (1973), 211.)

24 L. H. Harper. Stirling behavior is asymptotically normal. Annals Math. Statist. 38 (1967), 410-414.

25 T.-X. He. Parametric Catalan numbers and Catalan triangles. Linear Alg. Applic. 438 (2013), 1467-1484.

26 T.-X. He and R. Sprugnoli. Sequence characterization of Riordan arrays. Discr. Math. 309 (2009), 3962-3974.

27 S. Karlin. Total positivity, volume one (Stanford University Press, 1968).

28 C. Krattenthaler. On the $q$-log-concavity of Gaussian binomial coefficients. Monatsh. Math. 107 (1989), 333-339.

29 P. Leroux. Reduced matrices and $q$-log-concavity properties of $q$-Stirling numbers. J. Combin. Theory A 54 (1990), 64-84.

30 E. H. Lieb. Concavity properties and a generating function for Stirling numbers. J. Combin. Theory 5 (1968), 203-206.

31 L. L. Liu and Y. Wang. On the log-convexity of combinatorial sequences. Adv. Appl. Math. 39 (2007), 453-476.

32 D. Merlini, D. G. Rogers, R. Sprugnoli and M. C. Verri. On some alternative characterizations of Riordan arrays. Can. J. Math. 49 (1997), 301-320.

33 D. G. Rogers. Pascal triangles, Catalan numbers and renewal arrays. Discr. Math. 22 (1978), 301-310.

34 B. E. Sagan. Log concave sequences of symmetric functions and analogs of the Jacobi-Trudi determinants. Trans. Am. Math. Soc. 329 (1992), 795-811.

35 L. W. Shapiro. A Catalan triangle. Discr. Math. 14 (1976), 83-90.

36 L. W. Shapiro, S. Getu, W.-J. Woan and L. C. Woodson. The Riordan group. Discrete Appl. Math. 34 (1991), 229-239.

37 R. Sprugnoli. Combinatorial sums through Riordan arrays. J. Geom. 101 (2011), 195-210.

38 R. P. Stanley. Log-concave and unimodal sequences in algebra, combinatorics, and geometry. Annals New York Acad. Sci. 576 (1989), 500-534.

39 X.-T. Su and Y. Wang. On the unimodality problems in Pascal triangle. Electron. J. Combin. 15 (2008), paper 113.

40 S. Tanny. On some numbers related to the Bell numbers. Can. Math. Bull. 17 (1975), 733-738.

41 Y. Wang and Y.-N. Yeh. Log-concavity and LC-positivity. J. Combin. Theory A 114 (2007), 195-210.

42 B.-X. Zhu. Log-convexity and strong $q$-log-convexity for some triangular arrays. Adv. Appl. Math. 50 (2013), 595-606.

43 B.-X. Zhu. Some positivities in certain triangular array. Proc. Am. Math. Soc. 142 (2014), $2943-2952$. 